\title{
Bilder von Krankheit und Gesundheit: Das Beispiel Aids
}

\begin{abstract}
Images of Health and Disease: the Example of HIV/AIDS. There are two phases in the history of HIV/AIDS in the 1980s and 1990s. In the first of them, which lasted until the mid-1980s, HIV/AIDS was constructed as a disease of the (sexual) other. The second phase began around 1985 when the focus of AIDS prevention programs gradually shifted from „risk groups“ to „risk behavior“. This transformation came along with a reframing of the sexually active individual as self-reliant and socially responsible. Furthermore, the emergence of the risk discourse was accompanied by an iconography of a healthy and athletic „prevention body“. In the 1990s it increasingly replaced the emaciated „AIDS body“ that had dominated in the early years.
\end{abstract}

\section{Einleitung}

Aids/HIV stellte eines der zentralen gesundheitspolitischen Themen der 1980er und 1990er Jahre dar. Vor diesem Hintergrund gehe ich im Folgenden der Frage nach, inwiefern sich damals Bilder kranker und gesunder Körper veränderten. Ich argumentiere, dass sich zwei Phasen erkennen lassen, die allerdings nicht scharf voneinander getrennt werden können. Die erste entspricht ungefähr dem Zeitraum von 1982 bis zur Mitte des Jahrzehnts. Nicht zuletzt unter Bezug auf ältere Vorstellungen über Seuchen entstand das ikonische Bild des kranken und ausgemergelten „Aidskörpers“. Die zweite Phase begann um 1985, als die für die Geschichte von Aids zentrale Verschiebung des Fokus von den „Risikogruppen“ zum „Risikoverhalten“ einsetzte. Vor allem ab den 1990er Jahren schob sich vor diesem Hintergrund der gesunde und sportliche „Präventionskörper“ zunehmend an die Stelle des Aidskörpers.

Während meine Beispiele aus Deutschland und vor allem aus der Schweiz stammen, können die Resultate auch für Abbildungen von Krankheit und Ge-

\footnotetext{
Anmerkung: Beim vorliegenden Kapitel handelt es sich um eine stark gekürzte und aktualisierte Version eines Artikels, der zuerst in der Zeitschrift Body Politics veröffentlicht wurde (Bänziger 2014).
}

Peter-Paul Bänziger, Universität Basel

Ә OpenAccess. () 2021 Peter-Paul Bänziger, publiziert von De Gruyter. (cc))BY-NC-SA Dieses Werk ist lizenziert unter einer Creative Commons Namensnennung - Nicht kommerziell - Weitergabe unter gleichen Bedingungen 4.0 International Lizenz. https://doi.org/10.1515/9783110713336-029 
sundheit im Kontext von Aids/HIV in anderen Teilen Mittel- und Nordwesteuropas und teilweise darüber hinaus Gültigkeit beanspruchen (vgl. etwa Roeßiger 2013; Studinka 2002). Das gilt insbesondere für die erste Phase (Gilman 1991; Pulver 1999). Einer der Gründe dafür ist, dass Deutschland und die Schweiz schon früh als „Modellfälle“ beschrieben, beziehungsweise die deutsche Kampagne „GIB AIDS KEINE CHANCE“ und stärker noch die schweizerische „STOP AIDS“-Kampagne als „best practices“ zur Nachahmung empfohlen wurden (Bänziger \& Çetin 2019; Rosenbrock et al. 1999; Voegtli 2016).

\section{Von Seuchenvergleichen zum Aidskörper}

In einem der ersten Artikel im deutschsprachigen Raum, in dem auf Phänomene hingewiesen wurde, die später als Aids zusammengefasst werden sollten, schrieb Jean Lindenmann, Professor für Immunologie und Virologie an der Universität Zürich, über „alte und neue Geschlechtskrankheiten“ (Lindenmann 1982). An Beispielen wie diesem zeigte die kultur- und geschichtswissenschaftliche Forschung zu Aids schon früh auf, wie die in der Anfangszeit von Medien, Wissenschaften und Politik geschürte Krisenstimmung an die alte Angst vor der Syphilis und anderen übertragbaren Krankheiten anknüpfte. „Wie die Pest“ titelte etwa der SPIEGEL im Jahr 1983, wenn auch in durchaus kritischer Absicht (Der Spiegel 1983b). Und kurze Zeit später erklärte er, dass die „Krankheit [...] mittlerweile häufiger als Pest und Pocken zusammen“ sei (Der Spiegel 1983a, S. 239). Zwar gab es auch damals schon verschiedene Stimmen, die vor voreiligen Dramatisierungen warnten, doch auch sie verwendeten nicht selten ähnliche Vergleiche: „Ob AIDS zur modernen Pest wird, wie einige Kulturpessimisten befürchten, bleibt vorerst abzuwarten“ (Ringger 1983).

Eine Entsprechung fand der verbale Bezug auf die Pest in zahlreichen Bildern aus jenem Zeitraum. Zur Illustration des ersten Artikels in der schweizerischen Boulevardzeitung SonntagsBlick wurde etwa ein Foto abgedruckt, das die nackte Brustpartie eines Mannes zeigt, unter dessen Schlüsselbein deutlich drei dunkle Punkte zu erkennen sind (Abb. 1). Wie Sander Gilman (Gilman 1991) argumentiert, verweisen solche Bilder auf die Ikonographie der Beulen-Pest oder der Syphilis. Auch das Wort „Seuche“ im Titel macht diesen Bezug deutlich, während die Bildlegende mit dem „Krebs“ auf ein weiteres zentrales Thema jener Zeit verweist: „Oft erstes Anzeichen von Aids: purpurfarbene Flecken auf der Haut - das KaposiSarkom, eine Art Hautkrebs“ (Thomi 1983, S. 91). Der auf diese Weise eingeführte, abgemagerte „Aidskörper“ wurde zum ikonischen Bild von Aids, und zwar nicht nur in den Medien. Noch in den 1990er Jahren taucht er auf Plakaten auf (Abb. 2 und 3 ). 

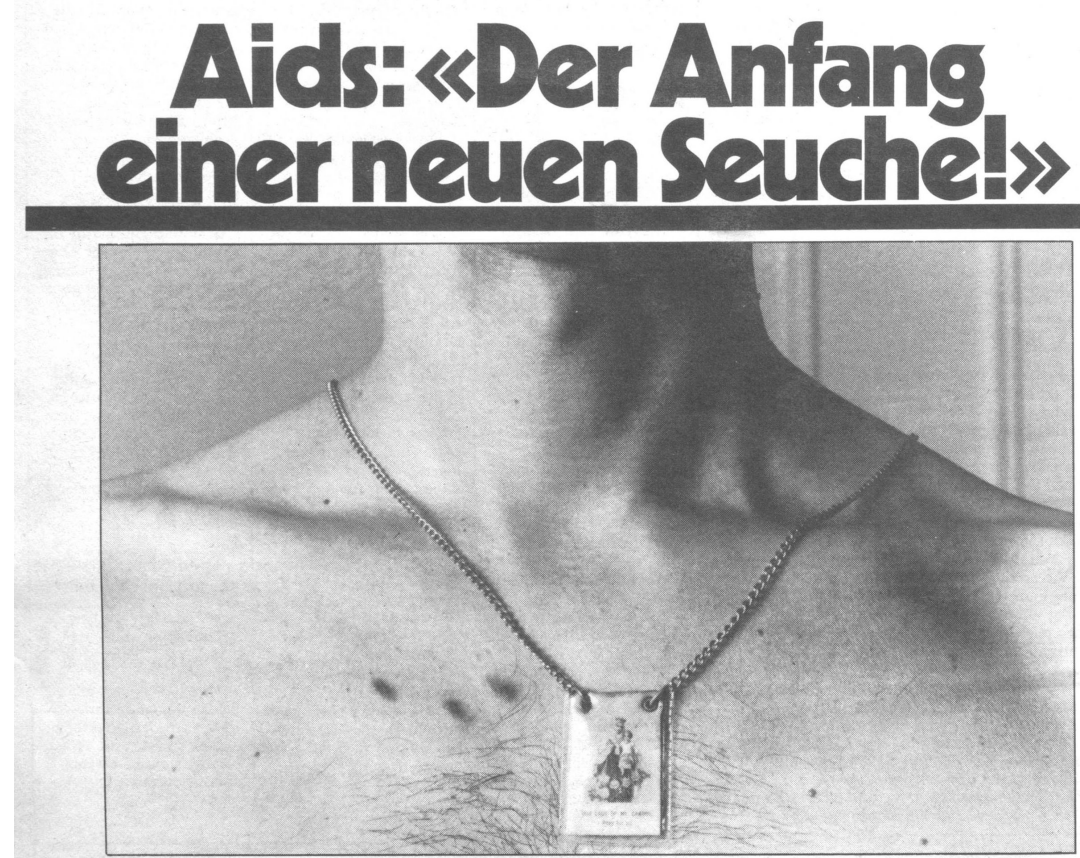

Oft erstes Anzeichen von Alds: purpurfarbene Flecken auf der Haut - das Kaposi-Sarkom, eine Art Hautkrebs.

Abb. 1: Foto aus dem schweizerischen SonntagsBlick (1983)

Der Aidskörper hatte verschiedene Gemeinsamkeiten mit der Figur des verelendeten „Junkie“ oder „Fixer“, der Ikone der Drogenthematik, des zweiten gesundheitspolitischen Brennpunktes jener Zeit. Beide Figuren wurden hauptsächlich in städtischen Umgebungen abgebildet. Beide waren deutlich durch ihre Krankheit beziehungsweise Sucht gezeichnet und wurden in der Regel als dieser passiv ausgesetzt dargestellt. „Nur in ihr Gesicht darf man nicht schauen: Die bleiche Haut ist von roten, aufgedunsenen Schwären überzogen. Sie hat Aids“, konnte man etwa in einem Bericht der ZEIT über die offene Drogenszene in Zürich lesen, der auf diese Weise eine direkte Verknüpfung zwischen Junkie- und Aidskörper herstellte (Sontheimer 1988). Nur selten hingegen wurden die Betroffenen im Kontext der Neuen Sozialen Bewegungen der 1970er und 1980er Jahre verortet, in denen sich viele von ihnen engagierten (vgl. Bänziger \& Çetin 2017). 


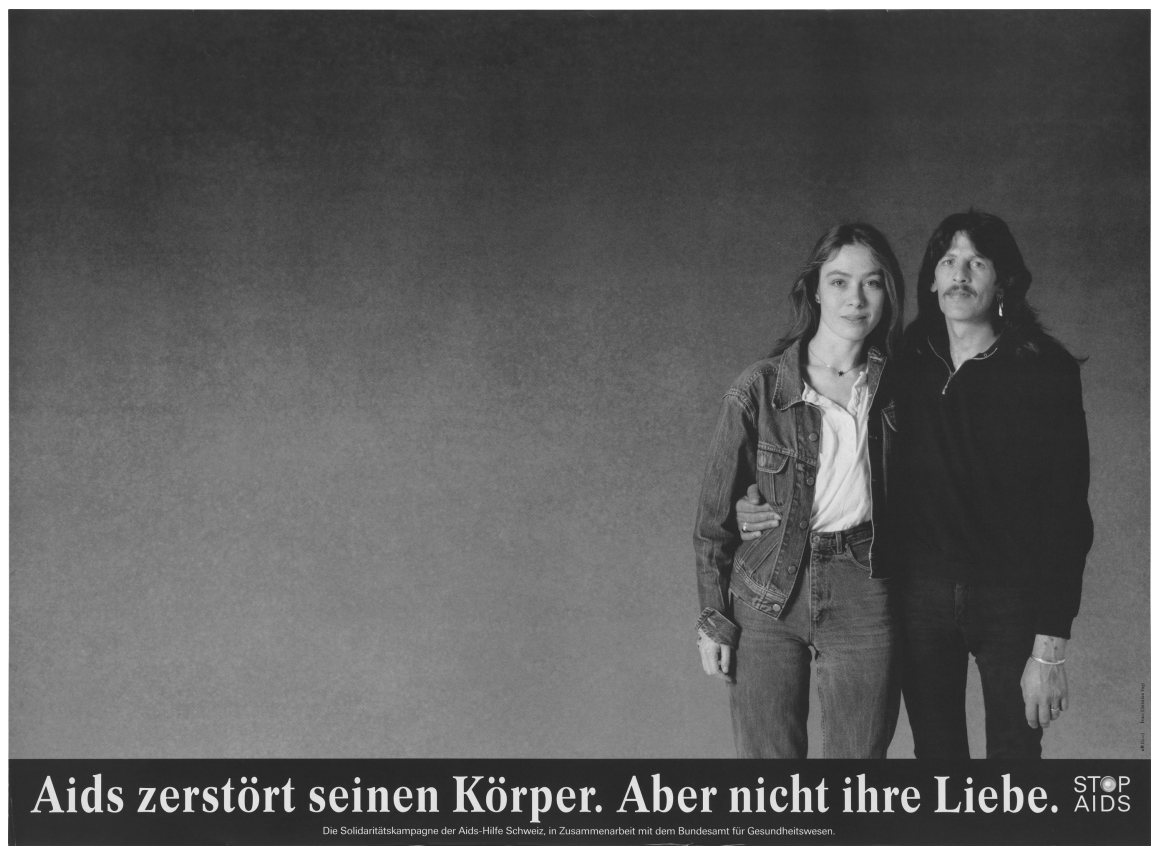

Abb. 2: Plakat der schweizerischen „STOP AIDS“-Kampagne (1990)

\section{Risikoverhalten: der Aufstieg eines neuen gesundheitspolitischen Regimes}

Der Aidskörper dominierte die Bildsprache während der 1980er Jahre. In der Aidspolitik hingegen kam es schon um die Mitte des Jahrzehnts zu einer weitreichenden Veränderung, als sich der Fokus von den „Risikogruppen“ zu den „Risikopraktiken“ beziehungsweise zum „Risikoverhalten“ zu verschieben begann. Neben der allmählichen Durchsetzung der These einer viralen Ursache von Aids ist dieser Wandel auch vor dem Hintergrund der gesundheitspolitischen Debatten über die „Prävention“ im 20. Jahrhundert zu betrachten (vgl. Lengwiler \& Madarász 2010; Thießen 2014). Vorausgegangen war ihm eine langjährige Auseinandersetzung um die zu wählenden Strategien, in der sich nicht zuletzt Aktivistinnen und Aktivisten aus dem Umfeld der Homosexuellen- und (weiteren) Betroffenenbewegungen vehement gegen Diskriminierungen und für die Freigabe der benötigten Ressourcen eingesetzt hatten (Beljan 2014, Kap. VI).

Zwar ist eine Risikogruppe über ähnliche Verhaltensweisen oder körperliche Dispositionen einer Anzahl von Personen definiert, in der Praxis wird diese Be- 


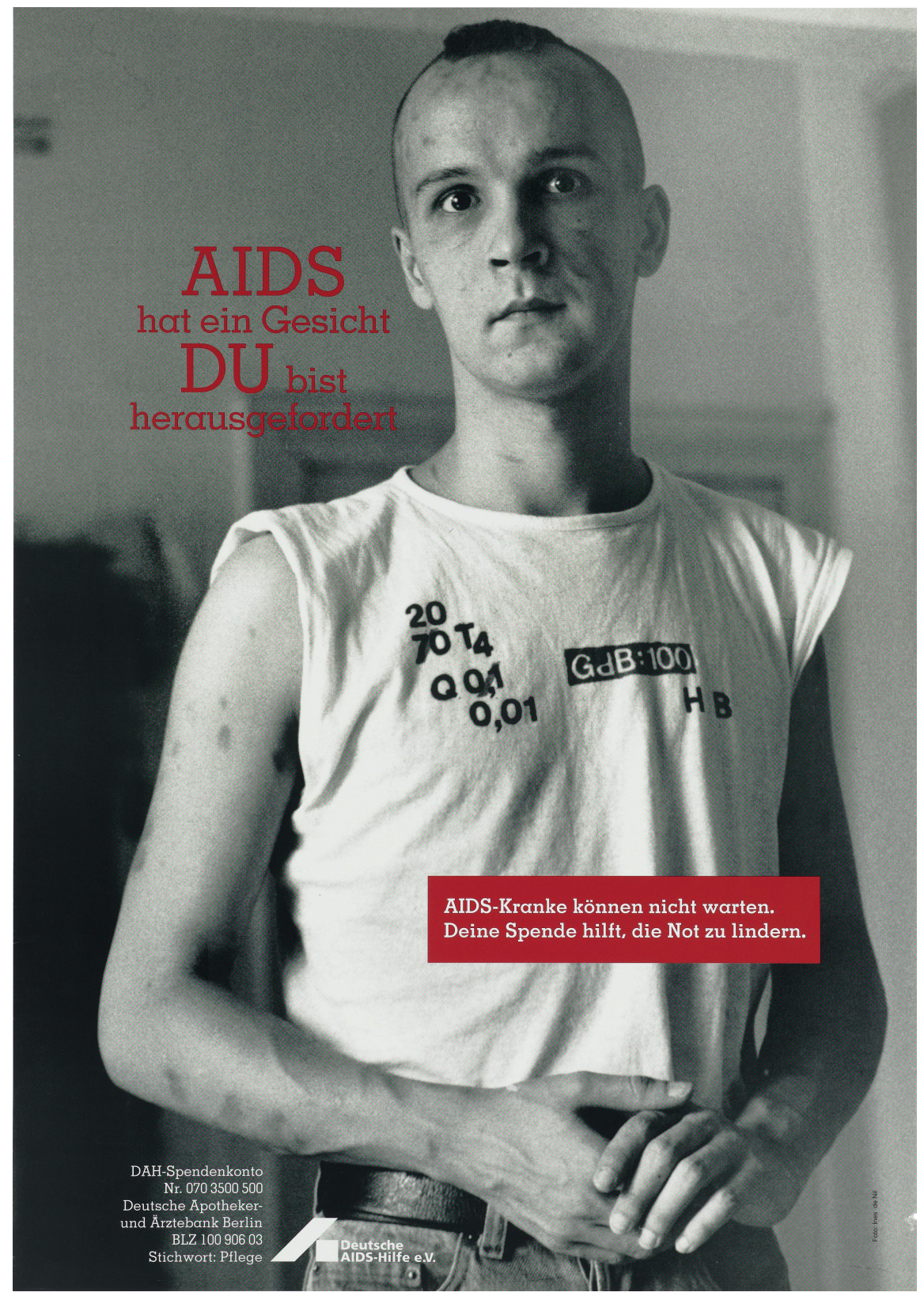

Abb. 3: Plakat der Deutschen Aids-Hilfe (1995) 
ziehung zwischen Kollektiv und Einzelpersonen jedoch oftmals umgekehrt: Von der (vermeintlichen) Zugehörigkeit zu einer gesellschaftlichen Gruppe wird auf das angeblich gemeinsame Verhalten der ihr zugerechneten Personen geschlossen. Zudem wird das Konzept der Risikogruppe oftmals überaus pauschal angewandt. Auch wenn es am Anfang durchaus hilfreich für die Aidsarbeit war, da es der Thematik die nötige Aufmerksamkeit unter den hauptsächlich Betroffenen verschaffte, zeigte sich diese Anschlussfähigkeit für diskriminierende Vorstellungen und Praktiken sehr bald. Ähnliches konnte man auch jüngst wieder bei den ,über 65-Jährigen“ während der SARS-CoV-2 Epidemie im Frühjahr 2020 beobachten, die pauschal als Risikogruppe bezeichnet und an manchen Orten vom öffentlichen Leben ausgeschlossen wurden. Man scheint aus der Aids-Krise nichts gelernt $\mathrm{zu}$ haben.

Das Konzept der Risikopraktiken erlaubte es damals, diskriminierende Tendenzen zu bekämpfen und differenzierter an die potentiell Betroffenen heranzutreten. Seine Etablierung erfolgte über einen längeren Zeitraum. Zunächst vergrößerte sich zunehmend die Zahl der Risikogruppen, da es ab Ende des Jahres 1984 immer schwieriger wurde, klare Grenzen zu ziehen. Im SPIEGEL stand Aids im Sommer 1984 zwar noch eindeutig als „Krankheit des Gettos und seiner Minderheiten“ in den Schlagzeilen, doch wurde nun darauf hingewiesen, dass möglicherweise die „Promiskuität“ ganz allgemein „das Vehikel der Seuche“ sei (Halter 1984, S. 131). Gegen Ende des Jahres wurde im Zusammenhang mit Homosexualität über „Safer Sex“ berichtet und die Forderung nach einer „Verhaltensänderung“ erhoben (Der Spiegel 1984, S. 258), die 1985 auf alle sexuell aktiven Personen ausgeweitet wurde. War zunächst noch von einer wenig konkreten „Allgemeingefährdung“ (Der Spiegel 1985, S. 235) die Rede, wurde im August ein Heft mit dem Titelthema „Aids und Liebe. Welches Risiko?“ veröffentlicht, als Titelbild ein sich küssendes heterosexuelles Paar. In verschiedenen Artikeln wurde darauf hingewiesen, dass die Zeit der Diskriminierung vorbei sei, weil Aids alle sexuell aktiven Personen betreffe.

Auf dieser Grundlage wurde der Fokus der Kampagnen und der Medienberichterstattung nun auf die Risikopraktiken verschoben: Schutz bot nicht mehr die Nichtzugehörigkeit zu einer Risikogruppe, sondern das Anpassen der je individuellen Handlungen an den aktuellen Stand des Wissens. So schrieben die Hannoveraner Sexualmediziner Wolfgang Müller und Klaus Pacharzina im SPIEGEL:

Es geht nicht um eine „neue Moral“, um „neuen Sex“, um Treue oder Monogamie. Wir Sexualmediziner sind gewiss nicht die Saubermänner der Nation. Dies ist keine Handlungsanleitung nur für „Risikogruppen“. [...] Gefragt ist verantwortliches Handeln, gefragt sind 
individuelle, freie Entscheidungen vor dem Hintergrund einer realen Gefahr (Müller \& Pacharzina 1985).

Zwar biete nur „Enthaltsamkeit“ vollkommene Sicherheit, doch da dies für die meisten nicht realistisch sei, gehe es vor allem darum, „das Risiko zu begrenzen“. Solche Anleitungen unterscheiden sich grundlegend von den Forderungen nach Verboten und „Notrecht“ (Moll 1984), die vereinzelt noch bis zum Ende der 1980er Jahre zu vernehmen waren. Im Frühjahr 1987 etwa beschloss die bayerische Regierung einen ganzen Katalog von Zwangsmitteln, vom Zwangstest bis zu drakonischen Strafen. Dass der SPIEGEL nun den Vorwurf erhob, Bayern wolle einen totalitären „Aids-Staat“ errichten (Der Spiegel 1987), erstaunt nicht. Das neue, auf Risikopraktiken ausgerichtete Regime hatte sich damals bereits durchgesetzt (vgl. Beljan 2014, S. 210 -211).

Aids sei geradezu die paradigmatische Epidemie eines neuen gesundheitspolitischen Regimes gewesen, argumentiert Delphine Moreau vor diesem Hintergrund (Moreau 2005). Da wichtigen Akteurinnen und Akteuren schon relativ früh klargeworden sei, dass das Immunschwächesyndrom in absehbarer Zeit nicht geheilt werden könne, hätten sie sich zunehmend darauf konzentriert, die HIV-Epidemie zu regulieren. An die Stelle des repressiven und überwachenden Zugriffs auf Gruppen oder Einzelpersonen sei die Information und statistische Kontrolle der Bevölkerung im Allgemeinen sowie einzelner Gruppen im Speziellen - die nun oftmals „Zielgruppen“ genannt wurden - getreten. Vor allem aber sollten die Kranken nicht mehr von den Gesunden oder Gefährdeten getrennt werden; alle sollten gleichermaßen lernen, sich risikominimierend zu verhalten. Eine Medikalisierung der Aids-Thematik hingegen wurde erst seit den 1990er Jahren breiter diskutiert. Dank neuer Möglichkeiten der Prä- und Postexpositionsbehandlung steht sie seit einigen Jahren gar im Zentrum der Aufmerksamkeit (vgl. Kippax \& Stephenson 2012).

Noch Ende 1984 hatte Friedhelm Farthmann, Gesundheitsminister in Nordrhein-Westfalen, in einem SPIEGEL-Interview erklärt, „daß der Mensch gegen Selbstschädigung nicht in allen Punkten geschützt werden“ könne und dass die „staatliche Verantwortung“ mit der Information jener Personen ende, „die bestimmte Sexualpraktiken“ pflegen (Farthmann 1984). Die Analyse der nationalen Präventionskampagnen zeigt jedoch, dass das Propagieren von risikominimierendem Verhalten und „Eigenverantwortung“ gerade nicht auf einfachen Appellen und dem Bereitstellen von Informationen beruhte, sondern auf einem ganzen Arsenal an Anleitungen zur Selbstführung, disziplinarischen „Maßnahmen“, Institutionen, wissenschaftlichen Expertisen u. a. m. (Bänziger 2015). Seit Aids sieht sich jede sexuell aktive Person mit der ultimativen Forderung konfrontiert, sich „richtig“ zu verhalten: verantwortlich im Umgang mit den eigenen wie auch den 
gesellschaftlichen „Ressourcen“ zu sein. Fragen nach den Ursachen für eine Infektion können nicht mehr mit einem tragischen „weil ich so bin“ oder einem unschuldigen „ich habe es nicht gewusst“ beantwortet werden.

\section{Vom Aids- zum Präventionskörper}

Die Verbreitung von Wissen über einzelne Praktiken war eine Bedingung für die Durchsetzung der neuen Aidspolitik, die Propagierung des Kondoms eine zweite. Hier stellte das Jahr 1987 einen wichtigen Wendepunkt dar: In Deutschland wie in der Schweiz setzten die nun anlaufenden Kampagnen an zentraler Stelle auf den „Gummi“. Neben dem spezifischen Wissen über sexuelle Praktiken wurde damit eine neue Norm aufgestellt, die man nicht ohne negative Sanktionen missachten durfte. Diese Problematik wurde schon durch die zeitgenössischen Sozialwissenschaften diskutiert, wenn etwa vermutet wurde, dass Befragte nicht mehr bereit waren, über unerwünschtes Verhalten wie den Verzicht auf das Kondom zu sprechen (vgl. Beljan 2015, S. $336 \mathrm{ff}$.). Solche Befunde sind jenen Stimmen gegenüberzustellen, die den Rückgang an Neuinfektionen direkt auf den Erfolg des neuen Präventionsregimes zurückführen. Viele Personen wussten zwar, wie sie sich schützen konnten. Die Frage hingegen, ob sie auch tatsächlich in diesem Sinne handelten, muss offen bleiben.

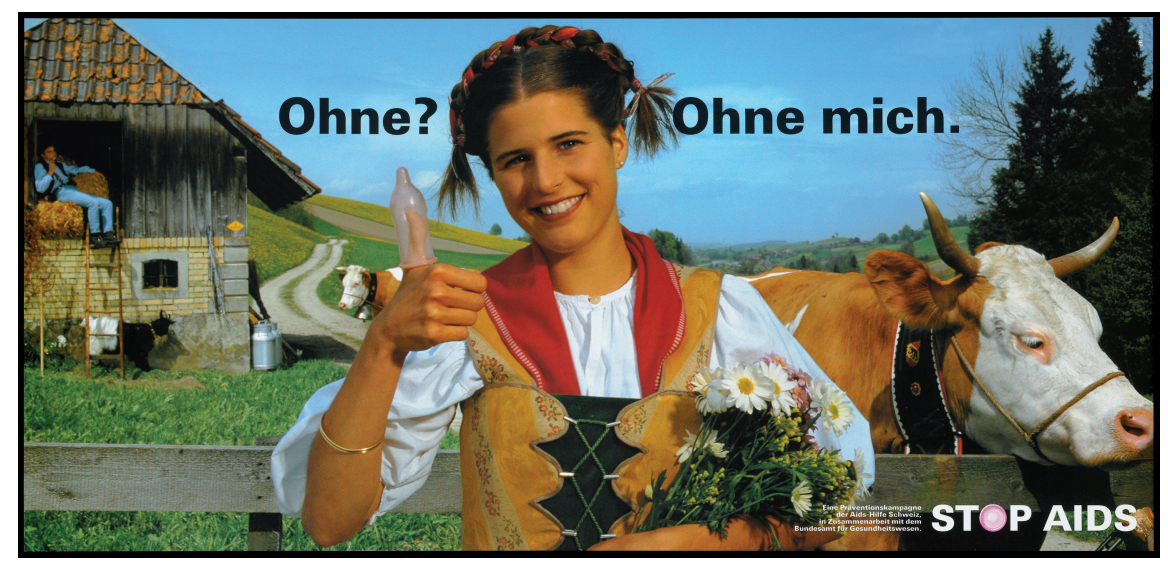

Abb. 4: Plakat der „STOP AIDS“-Kampagne, Schweiz (1992) (CC BY-NC 4.0)

Nicht zuletzt im Zusammenhang mit der Propagierung des Kondoms waren ab den frühen 1990er Jahren vermehrt Bilder „gesunder“ Körper zu sehen. Ein Blick auf die Plakate der schweizerischen „STOP AIDS“-Kampagne macht deutlich, wie 
nun eine neue Norm, jene des „Präventionskörpers“, den „Aidskörper“ der 1980er Jahre zunehmend in den Hintergrund drängte. Besonders signifikant ist ein Plakat aus dem Jahr 1992 (Abb. 4). Als Referenz für den Präventionskörper war die hier abgebildete bäuerliche Welt geradezu prädestiniert: Bäuerinnen und Bauern konnten als Menschen dargestellt werden, die sich durch die Arbeit in der Natur gesund erhalten, nicht nur in ökonomischer Hinsicht für sich selbst sorgen können und auch räumlich weit entfernt vom meist urbanen Umfeld von Aidskranken und Junkies leben.

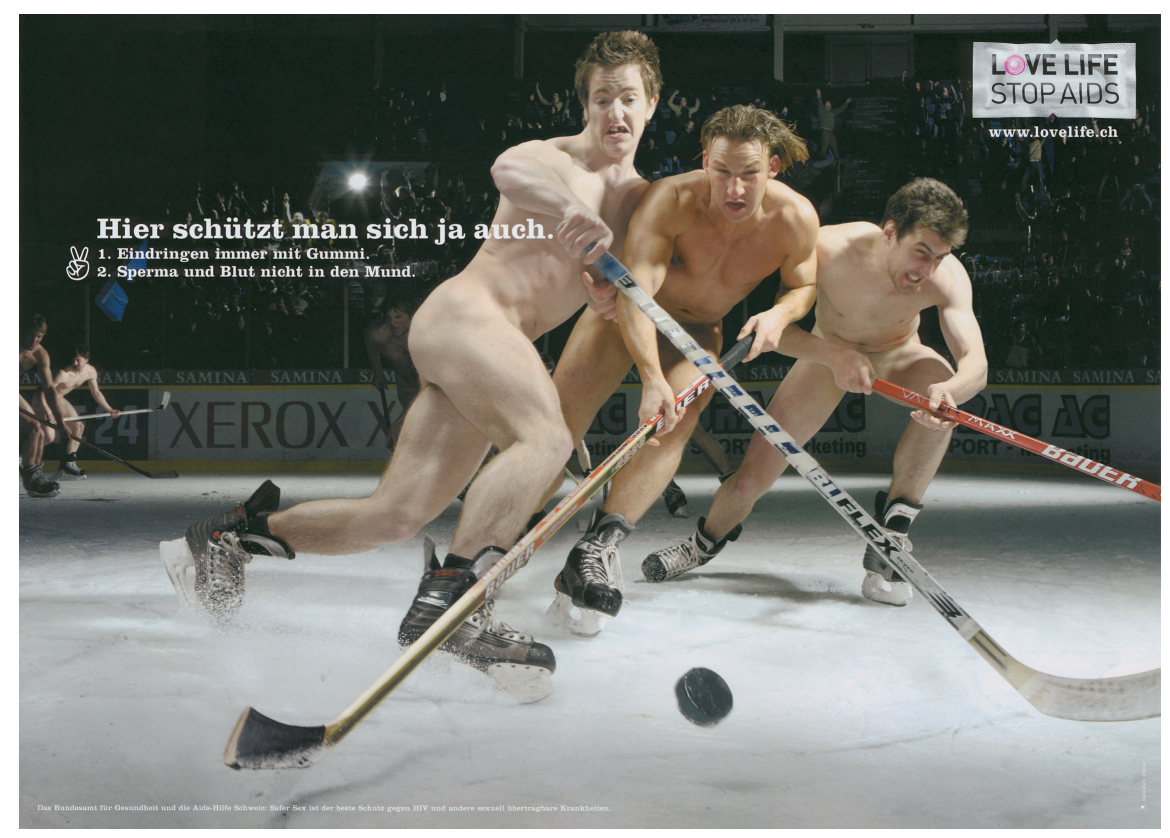

Abb. 5: Plakat der „STOP AIDS“-Kampagne, Schweiz (2006), Schweizerisches Sozialarchiv

Diese Bildsprache blieb in den folgenden Jahren zentral. Auf einem Plakat aus dem Jahr 2006 beispielsweise sind zwei unbekleidete Frauen beim Fechten zu sehen, auf einem anderen drei Eishockey spielende Männer, die lediglich Schlittschuhe tragen (Abb. 5). Der sportliche, attraktive Körper, so wird hier suggeriert, ist nicht jener des mit HIV infizierten oder an Aids erkrankten Mitmenschen, sondern jener des sich selbst schützenden Subjekts. Damit weist der Präventionskörper über die Aids-Thematik hinaus: Er ist nicht zuletzt im Kontext des Aufstiegs des fitten und sich selbst gut führenden Konsum- und Sexkörpers seit den 1980er Jahren (dazu Duttweiler 2004; Graf 2013; Wellmann 2015) zu betrachten. Zugleich konnte die durch die (implizite) Gegenüberstellung von Aids- 
und Präventionskörper behauptete Sichtbarkeit von Aids aber auch Grund für eine trügerische Sicherheit sein (Bänziger 2010, S. 204-205). Im Jahr 2000 reagierte die schweizerische „STOP AIDS“-Kampagne auf diese Problematik mit einem Plakat, auf welchem einem an der Ikonographie des Aidskörpers orientierten Bild eine herkömmliche Figur hegemonialer Männlichkeit - mit Hornbrille und Krawatte - gegenübergestellt wird, und nicht der fitte Präventionskörper (Abb. 6).

Sportlichen Personen, die mit HIV leben, begegnete man hingegen auch nach der Jahrtausendwende noch vergleichsweise selten. In einem Artikel der ZEIT Online zum Weltaidstag 2010 beispielsweise heißt es: „Als Klaus Wittke 1994 von seiner HIV-Infektion erfährt, gibt ihm sein Arzt noch wenige Jahre zu leben. Dass er nun, 16 Jahre später, einen Alltag ohne große Einschränkungen hat, dass er sogar Höchstleistungen bringt, ist für ihn ein kleines Wunder. “Im weiteren Text kommt unter anderen Jürgen Rockstroh, Infektiologe an der Universitätsklinik Bonn und Präsident der Deutschen Aids-Gesellschaft, zu Wort: „Erste Zwischenanalysen zeigen, dass das Immunsystem sich unter einer sportlichen Belastung wie einem Marathon positiv entwickelt“ (Blaschke 2010). Solche Texte zeigen, dass der Aidskörper auch in den Medien einem komplexeren Bild Platz machte - vor allem wenn es sich um Berichte über Menschen aus Europa und Nordamerika handelte, wo dank der neuen Therapiemöglichkeiten und starker Gesundheitssysteme die Lebensqualität auch bei einer Infektion mit HIV hoch bleiben kann.

\section{Schluss: Über die Erfolgserzählung hinaus}

Die hier vorgeschlagene Perspektive hilft, die Geschichte von Aids nicht einseitig als Erfolgsgeschichte zu erzählen (so etwa bei Tümmers 2017): Was einerseits zweifellos zur zahlenmäßigen Reduktion der Neuinfektionen beitrug, war andererseits mit spezifischen Forderungen an Körper und Subjekte verbunden. „Statt wohliger Lebenslust zu frönen, sollten wir ein Volk freudloser Aerobiner, Fitnesser und Weightwatcher sein, ansonsten lauert jetzt auch noch AIDS, um den Menschen ihre bösen Gelüste zu vergällen“, kritisierte ein Kommentator schon Mitte der 1980er Jahre (Jenny 1984). Freudlos mussten diese Körperpraktiken allerdings nicht unbedingt sein. Im Gegenteil: Die Norm des Präventionskörpers war gerade auch deshalb so erfolgreich, weil sie als lustvoll inszeniert und wohl auch erlebt wurde. Symbol dafür ist die „Safer Sex“-Kampagne, deren Ziel insbesondere darin bestand, das Überstreifen des Kondoms als Teil des sexuellen Spiels anstatt als dessen Unterbrechung darzustellen.

Zugleich brachte die neue Norm auch neues Diskriminierungspotential mit sich: Wenn alle fit und gesund sind, fallen jene umso mehr auf, die diesem Bild 


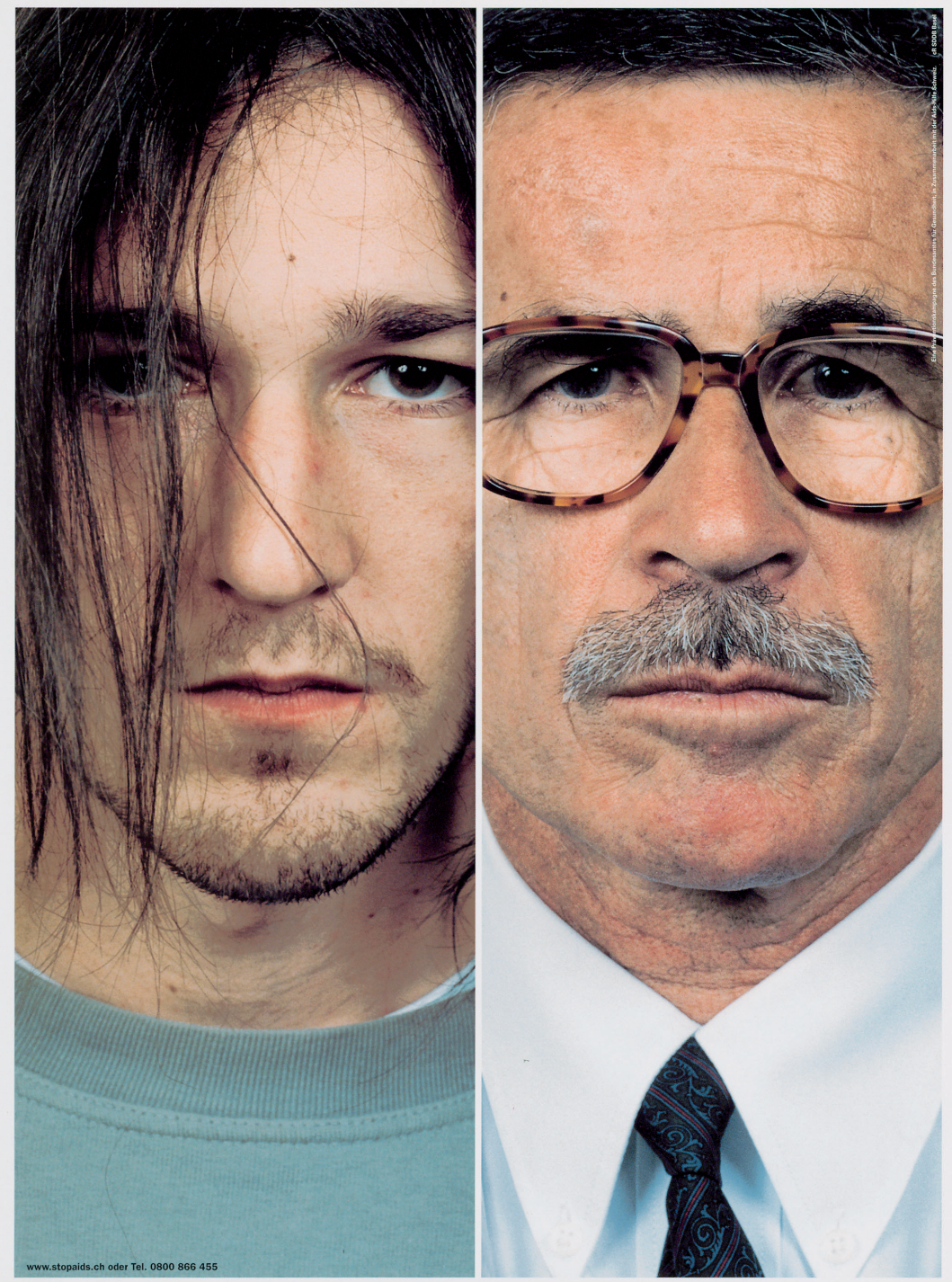

DER LINKS HAT WAHRSCHEINLICH NICHT. DER RECHTS HAT VIELLEICHT.

Man sieht jemandem nicht an, ob er sich mit dem HI-Virus infiziert hat. Wenn man eine neue Bekanntschaft macht, ist es vernunnftig, konsequent das Präservativ zu benutzen. Sagen Sie einfach.

Abb. 6: Plakat der „STOP AIDS“-Kampagne, Schweiz 2000. Schweizerische Nationalbibliothek, Graphische Sammlung: Plakatsammlung (cR Werbeagentur Basel) 
nicht entsprechen können oder wollen. Das Argument, dass sie offensichtlich keine Verantwortung für sich selbst wie für die Gemeinschaft übernehmen könnten, ist dann angesichts der Aufwertung der Eigenverantwortung in den vergangenen Jahrzehnten schnell zur Hand, um Kontrolle und Zwang zu legitimieren. Wie schon zu Beginn der Aids-Krise (vgl. Gilman 1991; Pulver 1999) stehen solche Körper weniger für die kranken, bemitleidenswerten Mitmenschen denn für einen selbstgewählten, problematischen Lebenswandel, für den sie die Verantwortung tragen müssen. Dass das nicht nur Spekulationen sind, hat sich während der SARS-CoV-2-Epidemie einmal mehr gezeigt. Zwar scheinen sich offene Diskriminierungen in Europa vor allem gegen Menschen mit angeblich „asiatischer“ Herkunft gerichtet zu haben, doch immer wieder waren auch Obdachlose betroffen (vgl. auch Anderson et al. 2020). Über ihre Lage schrieb ein Gassenarbeiter aus Zürich im Juni 2020:

„Viele Leute bieten Hilfe an, die Medien berichten über ihre Situation, es ist den Menschen nicht egal, was mit ihnen passiert. Andererseits leben wir in einer Zeit, in der alle zusammenzucken, wenn jemand nur hüstelt. Da geht man erst recht auf Distanz zu Menschen, die das Stigma von mangelnder Hygiene, Verwahrlosung und Krankheit tragen“(Brunner 2020).

\section{Literatur}

Anderson, Sage/Blok, Gemma/Fabian, Louise (2020): „Marginalization and Space in Times of COVID-19. Lockdown Report of the HERA Project: Governing the Narcotic City“. Kulturwissenschaftliches Institut Essen. https://narcotic.city/news/gonaci-lockdownreport/, besucht am 30.6.2020.

Bänziger, Peter-Paul (2010): Sex als Problem. Körper und Intimbeziehungen in Briefen an die „Liebe Marta“. Frankfurt am Main \& New York: Campus.

Bänziger, Peter-Paul (2014): „Vom Seuchen- zum Präventionskörper? Aids und Körperpolitik in der BRD und der Schweiz in den 1980er Jahren“. In: Body Politics 2(3), S. 179-214.

Bänziger, Peter-Paul (2015): „ExpertInnen statt AktivistInnen: Der Entpolitisierungsdiskurs in der Aids-Arbeit der achtziger Jahre“. In: Pascal Eitler/Jens Elberfeld (Hrsg.): Eine Zeitgeschichte des Selbst. Bielefeld: Transcript, S. 261-277.

Bänziger, Peter-Paul/Çetin, Zülfukar (2017): „Die Normalisierung eines Ausnahmezustands? Geschichten der Aids- und der Drogenthematik in der Bundesrepublik Deutschland seit den 1980er Jahren“. In: Hannah Ahlheim (Hrsg.): Gewalt, Zurichtung, Befreiung? Körperliche „Ausnahmezustände“ 1880-2000. Göttingen: Wallstein, S. 117-140.

Bänziger, Peter-Paul/Çetin, Zülfukar (2019): „Jenseits des Modellfalls. Erzählungen über Aids und HIV in Europa - eine Einleitung“. In: Peter-Paul Bänziger/Zülfukar Çetin (Hrsg.): Aids und HIV in der Türkei. Geschichten und Perspektiven einer emanzipatorischen Gesundheitspolitik. Gießen: Psychosozial-Verlag, S. 9-34.

Beljan, Magdalena (2014): Rosa Zeiten. Eine Geschichte der Subjektivierung männlicher Homosexualität in den 1970er und 1980er Jahren der BRD. Bielefeld: Transcript. 
Beljan, Magdalena (2015): „,Unlust bei der Lust?`Aids, HIV \& Sexualität in der BRD“. In: Peter-Paul Bänziger/Magdalena Beljan/Franz X. Eder/Pascal Eitler (Hrsg.): Sexuelle Revolution? Zur Geschichte der Sexualität im deutschsprachigen Raum seit den 1960er Jahren. Bielefeld: Transcript, S. 323-345.

Blaschke, Ronnie (2010): „Aids? Sport? Ja, klar!“. Zeit Online. https://www.zeit.de/sport/201012/aids-hiv-sport-schwimmen-positeidon, besucht am 30.6.2020.

Brunner, Raphael (2020): „,Noch einsamer als sonst‘. Obdachlose in Corona-Zeiten“. Beobachter Online. https://www.beobachter.ch/gesellschaft/obdachlose-corona-zeitennoch-einsamer-als-sonst, besucht am 30.6.2020.

Der Spiegel (1983a): „Lange Lunte“. Der Spiegel 47 vom 21.11.1983, S. 239-243.

Der Spiegel (1983b): „Wie die Pest“. Der Spiegel 28 vom 11.7.1983, S. 146-147.

Der Spiegel (1984): „Sprung nach vorn“. Der Spiegel 47 vom 12.11.1984, S. 255-261.

Der Spiegel (1985): „Harte Gegner“. Der Spiegel 18 vom 29.4.1985, S. 235-238.

Der Spiegel (1987): „,Wollen wir den Aids-Staat?‘ Bayerns Linie: Zwangstest, Berufsverbot, Ausweisung“. Der Spiegel 10 vom 2.3.1987, S. 30.

Duttweiler, Stefanie (2004): „Ein völlig neuer Mensch werden - Aktuelle Körpertechnologien als Medien der Subjektivierung“. In: Karl Brunner/Daniela Hammer-Tugendhat/Andrea Griesebner (Hrsg.): Verkörperte Differenzen. Wien: Turia \& Kant, S. 130-146.

Farthmann, Friedhelm (1984): „,Das ist eine schreckliche Vision‘. SPIEGEL-Interview mit NRW-Gesundheitsminister Friedhelm Farthmann“. Der Spiegel 45 vom 5.11.1984, S. $117-118$.

Gilman, Sander L. (1991): „AIDS and Syphilis: The Iconography of Disease“. In: Douglas Crimp (Hrsg.): AIDS: Cultural Analysis, Cultural Activism. Cambridge: MIT Press, S. 87-107.

Graf, Simon (2013): „Leistungsfähig, attraktiv, erfolgreich, jung und gesund: Der fitte Körper in post-fordistischen Verhältnissen“. In: Body Politics 1(1), S. 139-157.

Halter, Hans (1984): „,Ich bin en Tunt, bin kernjesund““. Der Spiegel 29 vom 16.7.1984, S. $130-134$.

Jenny, Reto G. (1984): „Zurück zu Sitte und Moral mit AIDS“. Tages-Anzeiger 187 vom 14.8.1984, S. 16.

Kippax, Susan/Stephenson, Niamh (2012): „Beyond the Distinction between Biomedical and Social Dimensions of HIV Prevention through the Lens of a Social Public Health“. In: American Journal of Public Health 102(5), S. 789-799.

Lengwiler, Martin/Madarász, Jeannette (Hrsg.) (2010): Das präventive Selbst. Eine Kulturgeschichte moderner Gesundheitspolitik. Bielefeld: Transcript.

Lindenmann, Jean (1982): „Alte und neue Geschlechtskrankheiten. Alarmierende Zunahme in jüngster Zeit“. Neue Zürcher Zeitung 190 vom 18.8.1982, S. 44.

Moll, Heinz (1984): „Nationalrat fordert: Mit Notrecht gegen AIDS“. SonntagsBlick 46 vom 11.11.1984, S. 18.

Moreau, Delphine (2005): „,Dispositifs de sécurité‘ et épidémie de sida“. In: Labyrinthe 22(3), S. $101-110$.

Müller, Wolfgang/Pacharzina, Klaus (1985): „,Das Risikopotential ist groß““. Der Spiegel 33 vom 12.8.1985, S. 152.

Pulver, Marco (1999): Tribut der Seuche oder: Seuchenmythen als Quelle sozialer Kalibrierung. Eine Rekonstruktion des AIDS-Diskurses vor dem Hintergrund von Studien zur Historizität des Seuchendispositivs. Frankfurt am Main: Peter Lang. 
Ringger, Heini (1983): „Wie viele AIDS-Patienten in der Schweiz?“. Tages-Anzeiger 153 vom 4.7.1983, S. 38.

Roeßiger, Susanne (2013): „Safer Sex und Solidarität. Die Sammlung internationaler Aidsplakate im Deutschen Hygiene-Museum“. In: Zeithistorische Forschungen/Studies in Contemporary History 10(3), S. 502-514.

Rosenbrock, Rolf/Schaeffer, Doris/Moers, Martin et al. (1999): Die Normalisierung von Aids in Westeuropa. Der Politik-Zyklus am Beispiel einer Infektionskrankheit. Berlin: Wissenschaftszentrum Berlin für Sozialforschung.

Sontheimer, Michael (1988): „Schweiz: Abschied von der Seuchenlehre“. Die Zeit 13 vom 25.3.1988, S. 14.

Studinka, Felix (Hrsg.) (2002): Visuelle Strategien gegen Aids. Zürich: Museum für Gestaltung \& Lars Müller Publishers.

Thießen, Malte (2014): „Seuchen im langen 20. Jahrhundert. Perspektiven für eine europäische Sozial- und Kulturgeschichte“. In: Malte Thießen (Hrsg.): Infiziertes Europa. Seuchen im langen 20. Jahrhundert. Berlin: De Gruyter, S. 7-28.

Thomi, Stefan (1983): „Aids: ,Der Anfang einer neuen Seuche!““. SonntagsBlick 21 vom 22.5.1983, S. $88-92$.

Tümmers, Henning (2017): Aids. Autopsie einer Bedrohung im geteilten Deutschland. Göttingen: Wallstein.

Voegtli, Michaël (2016): Une cause modèle. La lutte contre le sida en Suisse (1982-2008). Lausanne: Éditions Antipodes.

Wellmann, Annika (2015): „Silber-Sex. Von der Pathologisierung zur Aktivierung des gealterten Geschlechtskörpers“. In: Peter-Paul Bänziger/Magdalena Beljan/Franz X. Eder/Pascal Eitler (Hrsg.): Sexuelle Revolution? Zur Geschichte der Sexualität im deutschsprachigen Raum seit den 1960er Jahren. Bielefeld: Transcript, S. 303-322. 